Review began 02/17/2022 Review ended 02/25/2022 Published 02/27/2022

๑) Copyright 2022

Mierke et al. This is an open access article distributed under the terms of the Creative Commons Attribution License CC-BY 4.0. which permits unrestricted use, distribution, and reproduction in any medium, provided the original author and source are credited.

\section{Effect of Preoperative Opiate Use on Outcomes After Posterior Lumbar Surgery}

\author{
Alex Mierke ${ }^{1}$, Omar Ramos ${ }^{1}$, Jun Chung ${ }^{2}$, Wayne K. Cheng ${ }^{3}$, Olumide Danisa ${ }^{2}$ \\ 1. Orthopaedic Surgery, Loma Linda University Medical Center, Loma Linda, USA 2. Orthopaedics, Loma Linda \\ University Medical Center, Loma Linda, USA 3. Orthopaedics, Jerry L. Pettis Veterans Affairs Medical Center, Loma \\ Linda, USA
}

Corresponding author: Omar Ramos , o.ramos.williams@gmail.com

\section{Abstract}

\section{Introduction}

The prescription opioid epidemic and widespread use of narcotic medications have introduced new challenges when treating patients undergoing spine surgery. Given the ubiquity of preoperative opioid consumption amongst patients undergoing spine surgery, further research is needed to characterize perioperative risks. Our goal is to compare outcomes following primary lumbar decompression, instrumentation, and fusion based on preoperative opioid prescriptions.

\section{Methods}

Patients older than 18 years of age who underwent a primary one- to two-level lumbar decompression, instrumentation, and fusion were included in the study. Patients with known malignancy, surgery involving three or more lumbar levels, current or previous use of neuromodulation, revision surgery, anterior or far lateral interbody fusions, acute fractures, or other concurrent procedures were excluded. Patients were divided into chronic opioid therapy (COT; over six months), acute opioid therapy (AOT; up to six months), and opiate-naïve groups. Opioid prescriptions, demographics, smoking status, readmission rates within one year, and reoperation rates within two years were recorded based on electronic medical record documentation.

\section{Results}

Out of 416 patients identified, 114 patients met the inclusion criteria. Thirty-eight patients (33.3\%) were on COT, 38 patients (33.3\%) were on AOT, and 38 patients (33.3\%) were opioid naïve preoperatively. Readmission rates within one year for COT, AOT, and opioid naïve patients were $34.2 \%, 26.3 \%$, and $10.5 \%$, respectively $(\mathrm{p}=0.047$ ). Reoperation rates within two years for COT, AOT, and opioid naïve patients were $34.2 \%, 15.8 \%$, and $13.2 \%$, respectively ( $\mathrm{p}=0.049$ ). We also found current or former smokers were more likely to be on AOT or COT than never smokers ( $78.4 \%$ vs. $57.1 \%$; $\mathrm{p}=0.017)$.

\section{Conclusion}

Long-term opiate use is associated with an increased risk for readmission within one year and revision within two years. Physicians should discuss the increased risks of readmission and revision surgery associated with lumbar decompression and fusion seen in patients on preoperative opioid therapy.

Categories: Pain Management, Orthopedics, Epidemiology/Public Health Keywords: spine, readmission, smoking, lumbar-fusion, opioids use

\section{Introduction}

In the United States, the widespread use of opioid medications has created significant challenges for clinicians and patients. According to the 2019 National Survey on Drug Use and Health, two million people were diagnosed with an opioid use disorder in 2018 [1]. In 2018, there were 67,367 drug overdose deaths in the United States, two-thirds $(46,802)$ of those were due to opioids [2].

Over the past five years, multiple database studies have highlighted important associations between preoperative opioid use and surgical outcomes. Preoperative opioid use is associated with increased length of stay, increased 90-day readmission rates, implant-related complications, infection rates, pulmonary insufficiency, and increased healthcare-related costs [3-5]. These studies point to the dangers of chronic opioid therapy and its impact on clinical outcomes.

Nearly all previous studies rely on large databases to collect clinical and patient-reported outcomes. While this provides well-powered studies, limitations of a database study include reliance on administrative claims, coding methodology, and the inability to exclude patients who were prescribed opioids for unrelated 
problems $[4,6]$. The purpose of this study is to continue to characterize the impact that chronic opioid use has on surgical outcomes following one- or two-level lumbar decompression and fusion.

\section{Materials And Methods}

After the Human Investigation Committee (Institutional Review Board - IRB) of Loma Linda University approved this study (IRB\#5190139), the authors performed a retrospective review of the medical records of all patients who underwent lumbar decompression and fusion at a tertiary referral center from 2013-2017. Inclusion criteria included patients older than 18 years of age who underwent a primary one- or two-level posterior decompression (laminectomy or laminotomy) with either posterolateral intertransverse arthrodesis (PLF), transforaminal lumbar interbody fusion (TLIF), or posterior lumbar interbody fusion (PLIF). Exclusion criteria included patients without at least two years of follow up, patients undergoing revision surgery, patients with known malignancy (neoplasm of the spine or other body sites), surgery involving three or more levels, patients with current or previous use of neuromodulation (dorsal root ganglion stimulator or spinal cord stimulator), patients who required iliac crest bone graft harvest during surgery, anterior (ALIF) or far lateral interbody fusions (DLIF), patients with acute fractures or patients undergoing other concurrent procedures.

The definition of chronic opioid therapy (COT) varies significantly in the literature [7,8]. A 2019 study by Oleisky et al. [9] reviewed six definitions of preoperative opioid use in the literature and found that continuous use over six months was most predictive of postoperative satisfaction and patient-reported outcomes. Therefore, we utilized this definition for COT in our study. Patients were divided into chronic opioid therapy (COT; over six months), acute opioid therapy (AOT; up to six months), and opioid-naïve groups based on preoperative opioid usage. Opioid type, dose, frequency, and route were converted to milligram morphine equivalent (MME) dose. Primary outcomes included visual analog scale (VAS) scores, nicotine use, readmission rates within one year, and reoperation rates within two years.

VAS is a common, single-unit outcome measure for leg and back pain. The original description involves a 10 $\mathrm{cm}$ line with the numbers 0-10 associated with increasing pain scores from "no pain" to "unbearable pain". VAS scores were recorded for back and leg pain prior to surgery as well as between 6-12 months postoperatively. Data is presented as a "delta" VAS (final - initial) with negative numbers associated with improvement in pain. Nicotine use was recorded as "current", "former", or "never" users based on smoking history documented in the electronic medical record (EMR). Any readmission or subsequent surgery was recorded along with the underlying reason as documented in the EMR.

Categorical data was compared using chi-squared tests, and continuous variables were compared using analysis of variance (ANOVA) tests. Statistical analysis was conducted using SPSS 21.0 (IBM Inc., Armonk, USA).

\section{Results}

Initial review of patient data yielded 416 patients who met inclusion criteria by undergoing a one to twolevel posterior decompression, instrumentation, and fusion via PLF, TLIF, or PLIF between 2013 and 2017. Of the 416 patients, 297 were excluded because they were associated with revision surgery, fractures, malignancy, three or more levels, anterior or far lateral interbody fusions, and/or other concurrent procedures. Two patients in each group were lost to follow-up (5.3\%; Figure 1). 


\section{Cureus}

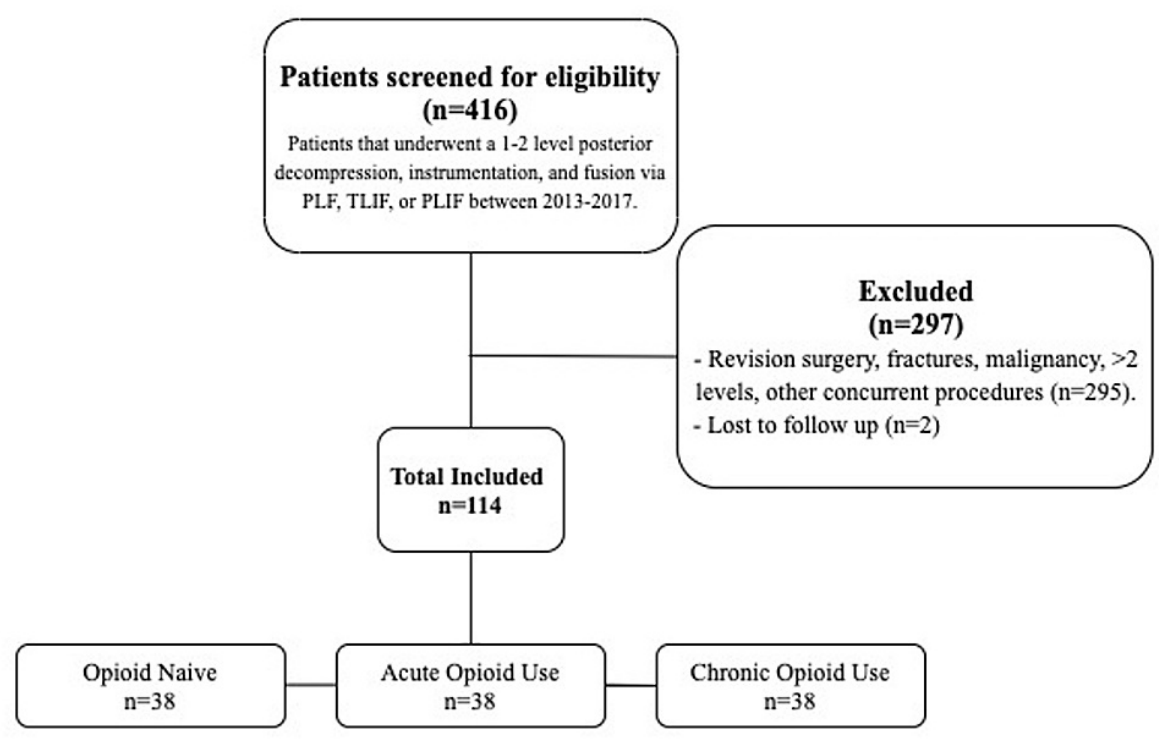

\section{FIGURE 1: Patient flow diagram}

PLF - posterolateral intertransverse arthrodesis; TLIF - transforaminal lumbar interbody fusion; PLIF - posterior lumbar interbody fusion

Table 1 shows the demographic characteristics of the 114 patients included in the final analysis. 


\section{Cureus}

\begin{tabular}{|c|c|c|c|c|}
\hline & Opioid naïve $(n=38)$ & Acute opioid use $(n=38)$ & Chronic opioid use $(n=38)$ & p-value \\
\hline Age at surgery: mean (SD) & $62.7(12.8)$ & $58.7(17.4)$ & $65.2(10.6)$ & 0.119 \\
\hline Gender: n (\%) & & & & 0.373 \\
\hline Female & $27(71.1 \%)$ & $22(57.9 \%)$ & $27(71.1 \%)$ & \\
\hline Male & $11(28.9 \%)$ & $16(42.1 \%)$ & $11(28.9 \%)$ & \\
\hline BMI: mean (SD) & $30.1(6.0)$ & $29.7(5.4)$ & $29.9(6.8)$ & 0.952 \\
\hline Levels: $\mathrm{n}(\%)$ & & & & 0.881 \\
\hline One level & $25(65.8 \%)$ & $21(55.3 \%)$ & $23(60.5 \%)$ & \\
\hline Two levels & $13(34.2 \%)$ & $17(44.7 \%)$ & $15(39.5 \%)$ & \\
\hline Smoking status: $\mathrm{n}(\%)$ & & & & 0.028 \\
\hline Never smoker & $27(71.1 \%)$ & $20(52.6 \%)$ & $16(42.1 \%)$ & \\
\hline Former smoker & $7(18.4 \%)$ & $11(28.9 \%)$ & $19(50 \%)$ & \\
\hline Current smoker & $4(10.5 \%)$ & $7(18.4 \%)$ & $3(7.9 \%)$ & \\
\hline Ethnicity: n (\%) & & & & 0.861 \\
\hline White & $25(65.8 \%)$ & $25(65.8 \%)$ & $28(73.7 \%)$ & \\
\hline Hispanic & $9(23.7 \%)$ & $10(26.3 \%)$ & $5(13.2 \%)$ & \\
\hline Asian & $2(5.3 \%)$ & $1(2.6 \%)$ & $2(5.3 \%)$ & \\
\hline African American & $2(5.3 \%)$ & $2(5.3 \%)$ & $2(5.3 \%)$ & \\
\hline Middle Eastern & $0(0 \%)$ & $0(0 \%)$ & $1(2.6 \%)$ & \\
\hline \multicolumn{5}{|l|}{ Visual analog scale (VAS) } \\
\hline Back pain & $-1.68(2.21)$ & $-2.18(2.53)$ & $-1.29(1.16)$ & 0.171 \\
\hline Leg pain & $-5.39(1.70)$ & $-4.61(2.14)$ & $-4.18(1.61)$ & 0.01 \\
\hline
\end{tabular}

\section{TABLE 1: Demographic characteristics}

Current or former nicotine users were more likely than non-users to be on acute or chronic opioid therapy (78.4\% vs. $57.1 \%$, $\mathrm{p}=0.017$; see Table 2 ).

\begin{tabular}{|c|c|c|c|}
\hline Smoking status: n (\%) & Opioid naïve & Acute or chronic opioid use & $\mathrm{p}$-value \\
\hline Never smoker & 27 (42.9\%) & $36(57.1 \%)$ & \multirow{2}{*}{0.017} \\
\hline Current or former smoker & $11(21.6 \%)$ & $40(78.4)$ & \\
\hline
\end{tabular}

TABLE 2: Smoking status and opioid use

The delta VAS scores for leg pain for COT, AOT, and opioid naïve patients were $-4.18,-4.61$, and -5.39 , respectively $(\mathrm{p}=0.016)$. The delta VAS scores for back pain for COT, AOT, and opioid naïve patients were $1.29,-2.18$, and -1.68 , respectively $(\mathrm{p}=0.171$ ). Patients who were opioid naïve received significantly more leg pain relief following surgery, but there was no significant absolute difference in back pain.

Given the known association between nicotine use and nociception, VAS scores were compared to nicotine use (Table 3). The delta VAS scores for back pain for never smokers, previous smokers, and current smokers were $-1.94,-1.73$, and -0.71 , respectively $(\mathrm{p}=0.004)$. Similarly, the delta VAS scores for leg pain for never smokers, previous smokers, and current smokers were $-4.97,-4.68$, and -3.79 , respectively $(\mathrm{p}=0.044)$. Due to this association, stratified analysis was performed to eliminate confounding variables. 


\section{Cureus}

\begin{tabular}{|c|c|c|c|c|}
\hline Delta VAS: mean (SD) & Never smoker $(n=63)$ & Former smoking $(n=37)$ & Current smoker $(n=14)$ & $p$-value \\
\hline Back pain & $-1.94(2.12)$ & $-1.73(1.73)$ & $-0.71(0.99)$ & 0.004 \\
\hline Leg pain & $-4.97(1.80)$ & $-4.68(2.31)$ & $-3.79(2.19)$ & 0.044 \\
\hline
\end{tabular}

\section{TABLE 3: Visual Analogue Scale and nicotine use}

VAS - Visual Analog Scale

Amongst never-smokers, the delta VAS scores for leg pain for COT, AOT, and opioid naïve patients were $4.56,-4.90$, and -5.26 , respectively ( $\mathrm{p}=0.53$ ). Amongst former smokers, delta VAS scores for leg pain for COT, AOT, and opioid naïve patients were $-3.95,-4.73$, and -6.57 , respectively $(\mathrm{p}=0.032)$. Amongst current smokers, delta VAS scores for leg pain for COT, AOT, and opioid naïve patients were $-3.67,-3.57$, and -4.25 , respectively ( $\mathrm{p}=0.897)$. Only former smokers had a statistically significant decrease in delta VAS scores for leg pain.

The readmission rate between opioid non-users, acute users, and chronic users was $10.5 \%, 26.3 \%$, and $34.2 \%$, respectively $(\mathrm{p}=0.047)$. Table 4 shows opioid usage versus readmission and reoperation rates.

\begin{tabular}{|c|c|c|c|c|}
\hline Readmission/reoperation rate: $\mathbf{n}(\%)$ & Opioid naïve & Acute opioid use & Chronic opioid use & p-value \\
\hline Readmission & $4(10.5 \%)$ & $10(26.3 \%)$ & $13(34.2 \%)$ & \multirow{2}{*}{0.047} \\
\hline No readmission & $34(89.5 \%)$ & $28(73.7 \%)$ & $25(65.8 \%)$ & \\
\hline Reoperation & $5(13.2 \%)$ & $6(15.8 \%)$ & $13(34.2 \%)$ & \multirow{2}{*}{0.049} \\
\hline No reoperatıon & $33(86.8 \%)$ & $32(84.2 \%)$ & $25(65.8 \%)$ & \\
\hline
\end{tabular}

TABLE 4: Opioid usage and readmission/reoperation rate

The reasons for readmission directly related to spine surgery included infection $(n=7,25.9 \%)$ and/or need for further surgery $(n=14,51.2 \%)$. Other reasons for admission that were indirectly related to spine surgery included stroke $(n=1,3.7 \%)$ gastrointestinal bleed $(n=1,3.7 \%)$, symptomatic anemia $(n=1,3.7 \%)$, deep vein thrombosis (DVT; $n=1,3.7 \%)$, perianal abscess $(n=1,3.7 \%)$ and pneumonia $(n=1,3.7 \%)$.

Causes for reoperation included infection $(n=3,12.5 \%)$, pseudarthrosis $(n=6,25 \%)$ adjacent segment disease $(n=3,12.5 \%)$ recurrent stenosis at another level $(n=7,29.2 \%)$ and/or implant migration $(n=5,20.8 \%)$.

\section{Discussion}

In the current study, the rate of readmission of opioid naïve, acute users, and chronic users was $10.5 \%$, $26.3 \%$, and $34.2 \%$, respectively ( $\mathrm{p}=0.047$ ). The rate of reoperation between opioid naive, acute users, and chronic users was $13.2 \%, 15.8 \%$, and $34.2 \%$, respectively $(\mathrm{p}=0.049)$. These findings are similar to those in recent studies, which also found a correlation between readmission or reoperation rate and history of opioid use $[4,5,10-13]$.

Increased readmission and reoperation rates ultimately lead to increased healthcare costs. In a prospective longitudinal registry study, Sivaganesan et al. [14] found that, among other variables, pre- and postoperative opioid use, readmission rates, and postoperative health care visits play an important role in the overall cost for elective spinal surgery. Jones et al. [15] found that the mean hospital revenue per day for geriatric patients with an opioid-related adverse drug event was $\$ 3,076$ less than patients without such an event. As it becomes more important to practice cost-conscious medicine, physicians should be aware of the increased risk of complications in opioid-dependent patients. Patients on chronic opioid therapy can be expected to place a larger burden on health care resources than their opioid naïve counterparts. Physicians are encouraged to maximize conservative management prior to surgery and to counsel patients preoperatively on their increased risk of readmission and reoperation.

Preoperative opioid use was associated with less improvement in postoperative leg pain but no difference in postoperative back pain relief (see Table 1). To eliminate nicotine use as a confounding variable, a stratified analysis was performed (Table 5). This analysis continued to demonstrate the negative association between 
preoperative opioid use and postoperative leg pain relief. This data is consistent with recent studies which demonstrate increased postoperative opioid requirements for patients using opioids preoperatively $[16,17]$. Given that patients on chronic preoperative opioid therapy perceive less pain improvement following surgery, clinicians operating on this subset of patients can expect worse outcome measures, regardless of technical ability.

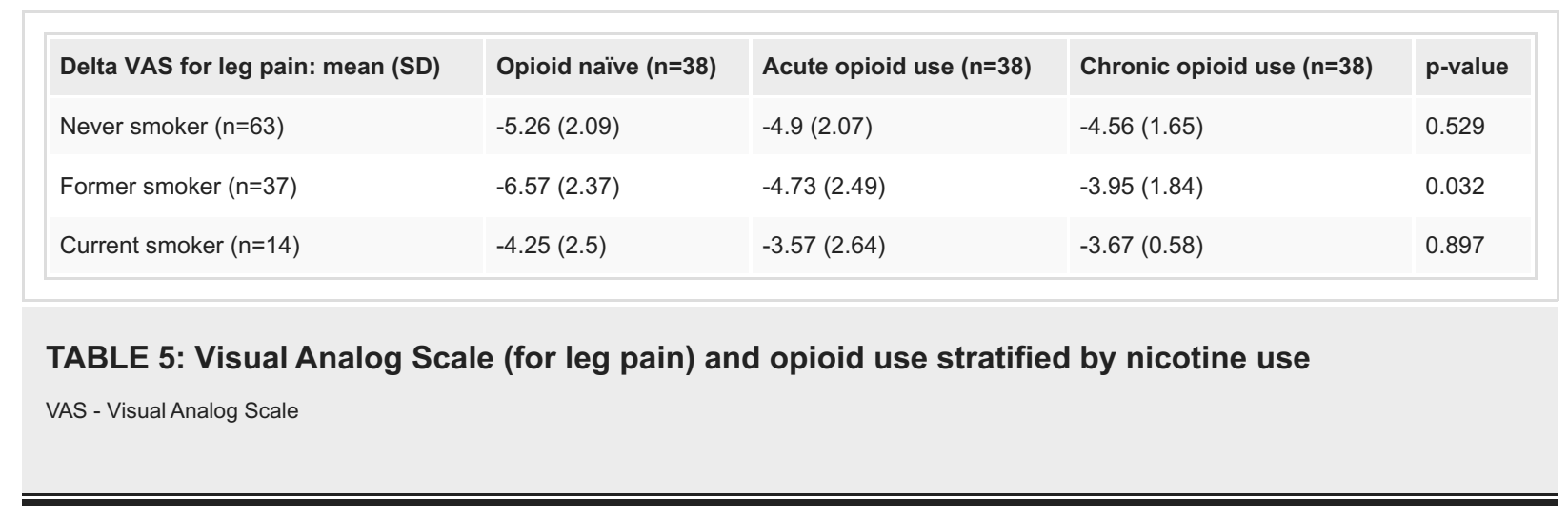

Current or former nicotine users were more likely than non-users to be on acute or chronic opioid therapy. This is supported by recent studies, which also found a correlation between nicotine and opioid dependence $[18,19]$. Previous studies have suggested that prolonged nicotine exposure is associated with structural damage to organ systems (osteoporosis, spinal degenerative disc disease, impaired bone, and wound healing), altered pain processing, personality disorders, and substance abuse $[18,20]$. Patients with a history of nicotine dependence are more likely to be on chronic opioid therapy and have diminished bone quality. Poor bone quality is associated with decreased screw pullout strength [21], pseudarthrosis [22], increased risk of hardware failure [23], adjacent level disc degeneration [24], and proximal junctional kyphosis [25], all of which can lead to reoperation or readmission. Physicians should be aware of these correlations and their impact on postoperative pain management requirements, readmission rates, and reoperation rates.

As the literature continues to demonstrate associations between preoperative opioid use and worsened outcomes, efforts to limit or wean preoperative opioids have been proposed. However, given the psychosocial complexity of chronic pain and opioid dependence, clinical studies are complicated by difficulties with randomization, noncompliance, and patient dropout. Current guidelines for opioid weaning, as described by Manchikanti et al., involve a 10\% reduction in opioids per week [26]. Furthermore, evidence from Lally et al. demonstrates that changing behavioral habits requires 12 weeks, with significant variation noted between individuals [27]. These studies indicate that opioid weaning trials can take a minimum of three months to implement, with high levels of relapse and noncompliance. Jain et al. reported fewer adverse events in patients that ceased opioid medications three months prior to surgery [4]. Nguyen et al. matched 41 patients on chronic opioid therapy (more than four weeks) with 41 opioid naïve patients and found that patients who weaned from opioids had similar patient-reported outcomes to opioid naïve patients [28]. More research is needed to establish a safe and effective preoperative opioid weaning program and the impact that such a plan would have on surgical and patient-reported outcome measures [29].

One limitation of this study is that it is a retrospective chart review that introduces possible errors in data input from practitioners and inaccuracy in patient-reported opioid dosages and durations. Furthermore, opioid dosage and duration were based on practitioner prescriptions but may not accurately reflect the actual prescriptions filled or medications taken. Also, we were not able to evaluate disease severity before surgery, which could affect the need for opioids before and after surgery. Recent database studies have taken advantage of insurance claims to monitor actual prescriptions filled, which we were unable to perform in our study. However, our study was able to look at more granular detail that International Classification of Diseases (ICD) 9 and 10 coding definitions lack, such as individual levels of surgery, approach, radiographic parameters, changes in patient-reported outcome measures such as VAS scores, and surgical/anesthesia notes. Loss to follow-up bias could have potentially affected the internal validity of our results. However, we found a loss of follow-up of only two patients in each group after applying the inclusion and exclusion criteria. This led to a loss of follow-up of $5.3 \%$ in each group. Previous studies have demonstrated that $<5 \%$ loss leads to little bias, while $>20 \%$ poses a serious threat to validity [30].

\section{Conclusions}

Chronic opioid use is associated with worse patient-reported outcome measures, increased risk for readmission within one year, and increased revision rate within two years following a primary posterior lumbar decompression, instrumentation, and fusion. Smokers are more likely to be chronic opioid users. Given the prevalence of opioid usage in the U.S. population, physicians should continue to educate acute and chronic opioid users about their increased risk of readmission and revision associated with lumbar decompression and fusion surgery. 


\section{Additional Information \\ Disclosures}

Human subjects: Consent was obtained or waived by all participants in this study. The Human Investigation Committee (IRB) of Loma Linda University issued approval IRB\#5200252. This retrospective chart review study involving human participants was in accordance with the ethical standards of the institutional and national research committee and with the 1964 Helsinki Declaration and its later amendments or comparable ethical standards. Animal subjects: All authors have confirmed that this study did not involve animal subjects or tissue. Conflicts of interest: In compliance with the ICMJE uniform disclosure form, all authors declare the following: Payment/services info: All authors have declared that no financial support was received from any organization for the submitted work. Financial relationships: Wayne K. Cheng declare(s) a grant from Medtronic, K2M, BIOMET, Ortho Fix, Nuvasive. Grants. Wayne K. Cheng declare(s) personal fees from Alphatec Spine, Depuy. Consulting fees. Olumide Danisa declare(s) a grant from Musculoskeletal Transplant Foundation, Globus Medical, Nuvasive, Medtronic . Grants. Olumide Danisa declare(s) non-financial support from NASS Resident/Fellowship Committee. Committee membership. Olumide Danisa declare(s) non-financial support from AAOS Medical Education Committee. Committee membership. Olumide Danisa declare(s) non-financial support from The Spine Journal. Editorial board. Other relationships: All authors have declared that there are no other relationships or activities that could appear to have influenced the submitted work.

\section{References}

1. U.S. Department of Health and Human Services (HHS) | What is the U.S. opioid epidemic? . (2019). Accessed: 12/19/21: http://www.hhs.gov/opioids/about-the-epidemic/index.html.

2. Drug overdose deaths in the United States, 1999-2018 . (2020). Accessed: 12/10/21: https://www.cdc.gov/nchs/data/databriefs/db356-h.pdf.

3. Cron DC, Englesbe MJ, Bolton CJ, et al.: Preoperative opioid use is independently associated with increased costs and worse outcomes after major abdominal surgery. Ann Surg. 2017, 265:695-701. 10.1097/SLA.0000000000001901

4. Jain N, Brock JL, Malik AT, Phillips FM, Khan SN: Prediction of complications, readmission, and revision surgery based on duration of preoperative opioid use: analysis of major joint replacement and lumbar fusion. J Bone Joint Surg Am. 2019, 101:384-91. 10.2106/JBJS.18.00502

5. Jain N, Phillips FM, Weaver T, Khan SN: Preoperative chronic opioid therapy: a risk factor for complications, readmission, continued opioid use and increased costs after one- and two-level posterior lumbar fusion. Spine. 2018, 43:1331-8. 10.1097/BRS.0000000000002609

6. Cook DJ, Kaskovich S, Pirkle S, Ho A, Conti Mica M, Shi L, Lee M: Benchmarks of Duration and Magnitude of Opioid Consumption After Common Spinal Procedures: A Database Analysis of 47,823 Patients. Spine. 2019, 44:1668-75. 10.1097/BRS.0000000000003141

7. Kha ST, Scheman J, Davin S, Benzel EC: The impact of preoperative chronic opioid therapy in patients undergoing decompression laminectomy of the lumbar spine. Spine. 2020, 45:438-43. 10.1097/BRS.0000000000003297

8. Lawrence JT, London N, Bohlman HH, Chin KR: Preoperative narcotic use as a predictor of clinical outcome: results following anterior cervical arthrodesis. Spine. 2008, 33:2074-8. 10.1097/BRS.0b013e3181809f07

9. Oleisky ER, Pennings JS, Hills J, et al.: Comparing different chronic preoperative opioid use definitions on outcomes after spine surgery. Spine J. 2019, 19:984-94. 10.1016/j.spinee.2018.12.014

10. Behrend C, Prasarn M, Coyne E, Horodyski M, Wright J, Rechtine GR: Smoking cessation related to improved patient-reported pain scores following spinal care. J Bone Joint Surg Am. 2012, 94:2161-6. 10.2106/JBJS.K.01598

11. Behrend CJ, Schönbach EM, Vaccaro AR, Coyne E, Prasarn ML, Rechtine GR: Maximum pain on visual analog scales in spinal disorders. Spine J. 2017, 17:1061-5. 10.1016/j.spinee.2016.11.017

12. Faour M, Anderson JT, Haas AR, Percy R, Woods ST, Ahn UM, Ahn NU: Prolonged preoperative opioid therapy associated with poor return to work rates after single-level cervical fusion for radiculopathy for patients receiving workers' compensation benefits. Spine. 2017, 42:104-10. 10.1097/BRS.0000000000001715

13. Weick J, Bawa H, Dirschl DR, Luu HH: Preoperative opioid use is associated with higher readmission and revision rates in total knee and total hip arthroplasty. J Bone Joint Surg Am. 2018, 100:1171-6. 10.2106/JBJS.17.01414

14. Sivaganesan A, Chotai S, Parker SL, McGirt MJ, Devin CJ: Drivers of variability in 90-day cost for elective laminectomy and fusion for lumbar degenerative disease. Neurosurgery. 2019, 84:1043-9. 10.1093/neuros/nyy264

15. Jones MR, Brovman EY, Novitch MB, Rao N, Urman RD: Potential opioid-related adverse events following spine surgery in elderly patients. Clin Neurol Neurosurg. 2019, 186:105550. 10.1016/j.clineuro.2019.105550

16. Dunn LK, Yerra S, Fang S, et al.: Incidence and risk factors for chronic postoperative opioid use after major spine surgery: a cross-sectional study with longitudinal outcome. Anesth Analg. 2018, 127:247-54. 10.1213/ANE.0000000000003338

17. Goesling J, Moser SE, Zaidi B, et al.: Trends and predictors of opioid use after total knee and total hip arthroplasty. Pain. 2016, 157:1259-65. 10.1097/j.pain.0000000000000516

18. Etcheson JI, Gwam CU, George NE, et al.: Opiate pain medication consumption in cigarette smokers following total hip arthroplasty. Joints. 2018, 6:157-60. 10.1055/s-0038-1673405

19. Hilliard PE, Waljee J, Moser S, et al.: Prevalence of preoperative opioid use and characteristics associated with opioid use among patients presenting for surgery. JAMA Surg. 2018, 153:929-37. 10.1001/jamasurg.2018.2102

20. John U, Hanke M, Meyer C, Völzke H, Baumeister SE, Alte D: Tobacco smoking in relation to pain in a 


\section{Cureus}

national general population survey. Prev Med. 2006, 43:477-81. 10.1016/j.ypmed.2006.07.005

21. Halvorson TL, Kelley LA, Thomas KA, Whitecloud TS 3rd, Cook SD: Effects of bone mineral density on pedicle screw fixation. Spine. 1994, 19:2415-20. 10.1097/00007632-199411000-00008

22. DeWald CJ, Stanley T: Instrumentation-related complications of multilevel fusions for adult spinal deformity patients over age 65: surgical considerations and treatment options in patients with poor bone quality. Spine. 2006, 31:144-51. 10.1097/01.brs.0000236893.65878.39

23. Rometsch E, Spruit M, Zigler JE, et al.: Screw-related complications after instrumentation of the osteoporotic spine: a systematic literature review with meta-analysis. Global Spine J. 2020, 10:69-88. $10.1177 / 2192568218818164$

24. Kim DK, Kim JY, Kim DY, Rhim SC, Yoon SH: Risk factors of proximal junctional kyphosis after multilevel fusion surgery: more than 2 years follow-up data. J Korean Neurosurg Soc. 2017, 60:174-80. 10.3340/jkns.2016.0707.014

25. Yagi M, Fujita N, Tsuji O, et al.: Low bone-mineral density is a significant risk for proximal junctional failure after surgical correction of adult spinal deformity: a propensity score-matched analysis. Spine. 2018, 43:485-91. 10.1097/BRS.0000000000002355

26. Manchikanti L, Kaye AM, Knezevic NN, et al.: Responsible, safe, and effective prescription of opioids for chronic non-cancer pain: American Society of Interventional Pain Physicians (ASIPP) guidelines. Pain Physician . 2017, 20:3-92.

27. Lally PvJCP, HW. Wardle, J: How are habits formed: modelling habit formation in the real world . Eur J Soc Psychol. 2010, 40:998-1009. 10.1002/ejsp.674

28. Nguyen AV, Ross E, Westra J, et al.: Opioid utilization in geriatric patients after operation for degenerative spine disease. J Neurosurg Anesthesiol. 2021, 33:315-22. 10.1097/ANA.0000000000000682

29. McAnally H: Rationale for and approach to preoperative opioid weaning: a preoperative optimization protocol. Perioper Med. 2017, 6:19. 10.1186/s13741-017-0079-y

30. Dettori JR: Loss to follow-up. Evid Based Spine Care J. 2011, 2:7-10. 10.1055/s-0030-1267080 